\title{
Occurrence of severe dengue in Rio de Janeiro: an ecological study
}

\author{
Gerusa Gibson ${ }^{[1]}$, Reinaldo Souza-Santos ${ }^{[1]}$, Alexandre San Pedro ${ }^{[1]}$, \\ Nildimar Alves Honório ${ }^{[2]}$ and Marilia Sá Carvalho ${ }^{[3]}$
}

[1]. Departamento de Endemias Samuel Pessoa, Escola Nacional de Saúde Pública, Fundação Oswaldo Cruz, Rio de Janeiro, RJ. [2]. Laboratório de Transmissores de Hematozoários, Fundação Oswaldo Cruz, Rio de Janeiro, RJ. [3]. Programa de Computação Científica, Fundação Oswaldo Cruz, Rio de Janeiro, RJ.

\begin{abstract}
Introduction: This study aimed to analyze the relationship between the incidence of severe dengue during the 2008 epidemic in Rio de Janeiro, Brazil, and socioeconomic indicators, as well as indicators of health service availability and previous circulation of the dengue virus serotype-3 (DENV-3). Methods: In this ecological study, the units of analysis were the districts of Rio de Janeiro. The data were incorporated into generalized linear models, and the incidence of severe dengue in each district was the outcome variable. Results: The districts with more cases of dengue fever in the 2001 epidemic and a higher percentage of residents who declared their skin color or race as black had higher incidence rates of severe dengue in the 2008 epidemic [incidence rate ratio $(\mathrm{IRR})=1.21 ; 95 \%$ confidence interval $\left({ }_{95 \%} \mathrm{CI}\right)=1.05-1.40$ and $\mathrm{IRR}=1.34 ;{ }_{95 \%} \mathrm{CI}=1.16-1.54$, respectively]. In contrast, the districts with Family Health Strategy (FHS) clinics were more likely to have lower incidence rates of severe dengue in the 2008 epidemic ( $I R R=0.81 ;{ }_{95 \%} \mathrm{CI}=0.70-0.93$ ). Conclusions: At the ecological level, our findings suggest the persistence of health inequalities in this region of Brazil that are possibly due to greater social vulnerability among the selfdeclared black population. Additionally, the protective effect of FHS clinics may be due to the ease of access to other levels of care in the health system or to a reduced vulnerability to dengue transmission that is afforded by local practices to promote health.
\end{abstract}

Keywords: Severe dengue. Health inequities. Self-declared skin color. Family Health Strategy. Epidemiology.

\section{INTRODUCTION}

In recent decades, dengue fever has gained prominence as one of the most important arboviral diseases in Brazil and worldwide because of the increasing number of people living in endemic areas as well as increases in severe clinical manifestations and lethality rates ${ }^{1}$.

Brazil accounts for more than $70 \%$ of notified cases in the Americas, and the City of Rio de Janeiro has become one of the most endemic cities in Brazil with a long history of dengue virus circulation ${ }^{2-5}$.

Successive epidemics have been reported in Rio de Janeiro, beginning in 1986 with the introduction of dengue virus serotype-1 (DENV-1) ${ }^{6}$. After an interval of four years, the city experienced an epidemic of dengue virus serotype-2 (DENV-2) from 1990-1991. During this epidemic, only a few cases of hemorrhagic fever were reported ${ }^{7}$. From 2001-2002, the largest epidemic to date, in terms of total cases, attributed to dengue virus serotype-3 (DENV-3) occurred in the city. This epidemic

Address to: Dra. Gerusa Gibson. Depto. de Endemias Samuel Pessoa/ ENSP/FIOCRUZ. R. Leopoldo Bulhões 1480/607, Manguinhos, 21041-210 Rio de Janeiro, RJ, Brasil.

e-mail: gibsonge@ensp.fiocruz.br

Phone: 5521 2598-2505; Fax: 5521 2598-2610

Received 17 September 2014

Accepted 6 November 2014 resulted in more than 288,000 notified cases with 1,831 cases of hemorrhagic fever and 91 death $^{8,9}$. Subsequently, the City of Rio de Janeiro was affected in 2008 by the largest dengue epidemic ever reported in terms of mortality. The 2008 epidemic was associated with a variant strain of the DENV-2 serotype, which was characterized by a greater proportion of severe cases in children compared with previous epidemics ${ }^{2,5,10,11}$.

No consensus is currently available on the determinants of a poor clinical prognosis in dengue patients; however, the excessive number of severe cases in Rio de Janeiro in 2008 may be explained by a combination of factors that range from proximal determinants, such as the genetic variant of the DENV-2 serotype, the immune status of the population, the supply and quality of health services, and the socioeconomic context from which the severe cases emerged ${ }^{2,4,5,11}$.

Therefore, this ecological study aimed to analyze the association between the occurrence of severe cases of dengue in the 2008 epidemic and socioeconomic indicators, as well as indicators of health service availability and previous circulation of the DENV-3 serotype.

\section{METHODS}

\section{Study area}

The study area was Rio de Janeiro, the capital of the State of Rio de Janeiro in the southeast region of Brazil. According to 2010 census data, the city has a population of $6,323,037$ 
inhabitants within an area of $1,182 \mathrm{~km}^{2}$ that is divided into 160 districts. These districts were the units of analysis in this ecological study.

\section{Data sources}

This study analyzed confirmed cases of severe dengue fever [dengue hemorrhagic fever (DHF); dengue shock syndrome (DSS); and dengue with complications (DCC)] that occurred in Rio de Janeiro in 2008. Data were collected from the National System for Notifiable Diseases [Sistema Nacional de Agravos de Notificação (SINAN)]. Duplicate records and inconsistencies were removed from the database, and the cases were categorized according to the district of residence.

In Brazil, the Ministry of Health has adopted the definitions for suspected and confirmed cases of dengue fever and hemorrhagic dengue fever that were proposed by the World Health Organization (WHO). However, because of difficulties in classifying severe cases of disease, an intermediate classification called dengue with complications [dengue com complicações (DCC)] was adopted in Brazil ${ }^{12}$. Dengue with complications is assigned to cases with clinical outcomes that do not completely meet the traditional WHO classification criteria for dengue hemorrhagic fever. Additionally, DCC may indicate cases in which the dengue fever rating is unsatisfactory given the severity of the clinical and laboratory manifestations. Therefore, DCC includes cases with at least one of the following clinical and laboratory changes: cardio-respiratory dysfunction, liver failure, massive gastrointestinal bleeding, neurological abnormalities, a leukocyte count equal to or less than $1,000 \mathrm{cells} / \mathrm{ml}$, a platelet count less than $20,000 / \mathrm{ml}$, pleural effusion, pericardial effusion or ascites. In addition, fatal dengue cases that do not meet the criteria for hemorrhagic fever may be classified as $\mathrm{DCC}^{12}$.

The data on health facilities were extracted from the National Register of Health Establishments [Cadastro Nacional de Estabelecimentos de Saúde (CNES)]. We selected only public health units and classified them according to type and location. The data used to construct the socioeconomic indicators were obtained from the 2010 census from the Brazilian Institute of Geography and Statistics [Instituto Brasileiro de Geografia e Estatistica (IBGE)]. The district populations for 2001 and 2008 (inter-census) were estimated based on the geometric growth model.

\section{Data analysis}

Using the available data from the 2010 census, we constructed 15 possible indicators related to the disease. The following indicators were calculated per district: the number of severe dengue cases in the 2001 epidemic (considered to be a proxy for the prior circulation of the DENV-3 serotype); the percentage of residents who declared their skin color or race as black; the proportion of permanent private domiciles that store rain water in cisterns; the proportion of permanent private domiciles that discharge domestic waste on empty lots or on the street; the ratio of the population within a given district to the district area in $\mathrm{km}^{2}$; the proportion of permanent private domiciles in substandard settlements; the proportion of residents who live in substandard settlements; the proportion of permanent domiciles in substandard settlements in which domestic waste is collected by open skip (waste is accumulated in a large dumpster without a lid, which is shared with the whole neighborhood and collected at time intervals that are usually longer than those of standard domestic waste collection service); the proportion of permanent private domiciles with no connection to the sewage system and no septic tank; the proportion of permanent private domiciles in which the head of the family has an income less than the Brazilian monthly minimum wage (approximately US\$290); the proportion of residents in permanent private domiciles with no exclusive bathroom available and no toilet; the proportion of permanent private domiciles in which domestic waste is collected by open skip; the proportion of permanent private domiciles in which the water supply is from a well or spring on the property; the number of primary health facilities; and the number of Family Health Strategy (FHS) clinics.

The Family Health Strategy program was designed to reorient the Brazilian health care model by implementing multidisciplinary teams in primary care units. These teams are responsible for monitoring families who reside in a particular geographical area, and they usually work in the community on initiatives for health promotion and prevention, including the rehabilitation of diseases and health disorders. The aim of the FHS is to achieve health assistance that is equitable and comprehensive $\mathrm{e}^{13,14,15}$.

Subsequently, the indicators were separated into groups according to subject (sanitation, income, health services and education) and a collinearity diagnostic was performed using the variance inflation factor (VIF) test (with a tolerance value greater than 10) to select variables for the model. The explanatory variables were tested separately in univariate models, and the variables with statistical significance were analyzed in multivariate models using forward selection logistic regression. The likelihood ratio test was used to assess the best-fit model. Only the results of the negative binomial model with the best fit are presented. Moran's index was used to measure the spatial autocorrelation of the residuals from the model. The analyses were performed using the $\mathrm{R}$ software environment ( $\mathrm{R}$ Development Core Team 2011; R Foundation for Statistical Computing, Vienna, Austria).

The distribution of the means and standard deviations of the explanatory variables was calculated for the overall set of districts; the values were subsequently standardized using the $\mathrm{Z}$-scale [(X-mean)/standard deviation]. Using this method, the magnitude of the change in the incidence rate ratio for severe dengue cases was compared for each predictive variable ${ }^{13,16}$.

The data were incorporated into a negative binomial regression model, which is commonly used for counting data and when the variance exceeds the mean ${ }^{17}$. Because the dependent variable (severe dengue cases) was influenced by the incidence rate of dengue fever, the analysis was adjusted for the number of dengue fever cases in 2008.

The dependent variable $Y_{i}$ for each district $\left({ }^{i=1,2,3 . ., 156)}\right.$ has an expected value of $\mu_{i}$ and a dispersion parameter $\theta$, which was used to capture the extra-variation in the data ${ }^{17}$. The overdispersion of error terms was tested using the poisgof function in the epicalc package, which was included in the 
$\mathrm{R}$ software program 2.11.1 ( $p$-value $=0.0082)$. The model was expressed as $\log (\mu)=\beta \chi+\varepsilon$, where $\chi_{i}$ is the standardized independent variable (with its associated regression coefficient, $\beta_{i}$ ) and $\varepsilon$ represents the error term ${ }^{17}$. The natural logarithm of the district population was included as the offset variable. The exponential of each $\beta_{i}$ regression coefficient provides the incidence rate ratio for each 1 -standard-deviation change in the corresponding independent variable.

\section{Ethical considerations}

All of the activities in this study were in compliance with the guidelines of the research ethics committee of the Escola Nacional de Saúde Pública, decision number 43/11.

\section{RESULTS}

In 2008, a total of 59,395 cases of dengue were reported in Rio de Janeiro, of which 12,620 cases were classified as DF, 5,082 as DCC, 621 as DHF, and 18 as DSS. A final classification was not available for 41,054 cases; therefore, approximately $31 \%$ of the total cases were classified. The age group that was most affected by all clinical forms of the disease were children and adolescents 6-15 years of age. Higher incidence rates were found in this age group compared with other age groups (Table 1).

Furthermore, according to the data provided by Sistema Nacional de Agravos de Notificação (SINAN), a total of 5,463 patients with dengue were hospitalized in Rio de Janeiro in 2008 , representing $9 \%$ of the 59,395 dengue cases reported in the city. The highest proportion of death was found in patients who were 6-15 years and those older than 60 years.

In the univariate analysis, two variables were significantly associated with the occurrence of severe dengue cases in the 2008 epidemic: the number of dengue fever cases per district in the epidemic year of 2001 and the percentage of residents who declared their skin color or race as black. The number of FHS clinics and the proportion of permanent domiciles in substandard settlements in which domestic waste was collected by open skip were inversely associated with the incidence of severe dengue, and these associations were significant. However, when these variables were combined in a multivariate model, the proportion of permanent domiciles in substandard settlements in which domestic waste was collected by open skip lost statistical significance as an explanatory factor and was therefore eliminated from the final model. Additionally, a direct association was observed between the number of dengue fever cases and the incidence of severe cases in 2008 (Table 2).

The districts with more cases of dengue during the 2001 epidemic (an epidemic attributed to the DENV-3 serotype) presented higher incidence rates of severe dengue during the 2008 epidemic, both in the univariate and multivariate models. Similarly, the districts with a higher percentage of residents who declared their skin color or race as black were more likely to have higher incidence rates of severe dengue in 2008 in both the univariate and multivariate models. In contrast, the districts with more FHS clinics were more likely to have lower incidence rates of severe dengue in the 2008 epidemic. All of the multivariate analyses were adjusted for the number of dengue fever cases in 2008 (Table 2). The incidence rates of dengue fever and severe dengue in 2008 for each district in Rio de Janeiro are shown in Figure 1 and Figure 2.

For each increment of one standard deviation in the mean number of dengue cases in 2001 (approximately from 166 to 263), the incidence rate of severe dengue in 2008 increases 1.21 times (assuming the other variables remained constant). Similarly, for each increment of one standard deviation in the mean percentage of residents who declared their skin color or race as black (approximately from $11.5 \%$ to $16 \%$ ), the incidence rate of severe dengue in 2008 increases 1.34 times. For each increment of one standard deviation in the mean number of FHS clinics (from 0.2 to 0.6 ), the incidence rate of severe dengue in 2008 was 0.2 times lower (Table 2 and Table 3 ).

TABLE 1 - The incidence of dengue according to age and clinical form in Rio de Janeiro, Brazil in 2008.

\begin{tabular}{|c|c|c|c|c|c|c|}
\hline $\begin{array}{l}\text { Age group } \\
\text { (years) }\end{array}$ & \multicolumn{2}{|c|}{ Dengue fever } & \multicolumn{2}{|c|}{ Severe dengue } & $\mathrm{NM}+$ & Total \\
\hline$\leq 5$ & 1,016 & 204.1 & 410 & 82.4 & 3,668 & 5,094 \\
\hline $6-15$ & 3,269 & 364.1 & 2,632 & 293.1 & 11,017 & 16,918 \\
\hline $16-20$ & 1,099 & 308.8 & 365 & 102.6 & 3,810 & 5,274 \\
\hline $51-60$ & 1,141 & 159.9 & 377 & 52.8 & 2,888 & 4,406 \\
\hline$>60$ & 804 & 90.8 & 387 & 43.7 & 1,903 & 3,094 \\
\hline Total & 12,620 & 204.8 & 5,721 & 92.9 & 41,054 & 59,395 \\
\hline
\end{tabular}

+ Number of missings: cases with missing classification. *Incidence per 100,000 inhabitants; +the number of case records in which the final classification was ignored or not completed; the cases classified as dengue hemorrhagic fever, dengue shock syndrome, and dengue with complications were considered to be severe infections. 
TABLE 2 - Parameters of the univariate and multivariate analyses and their respective incidence rate ratios of severe dengue in Rio de Janeiro, Brazil in 2008.

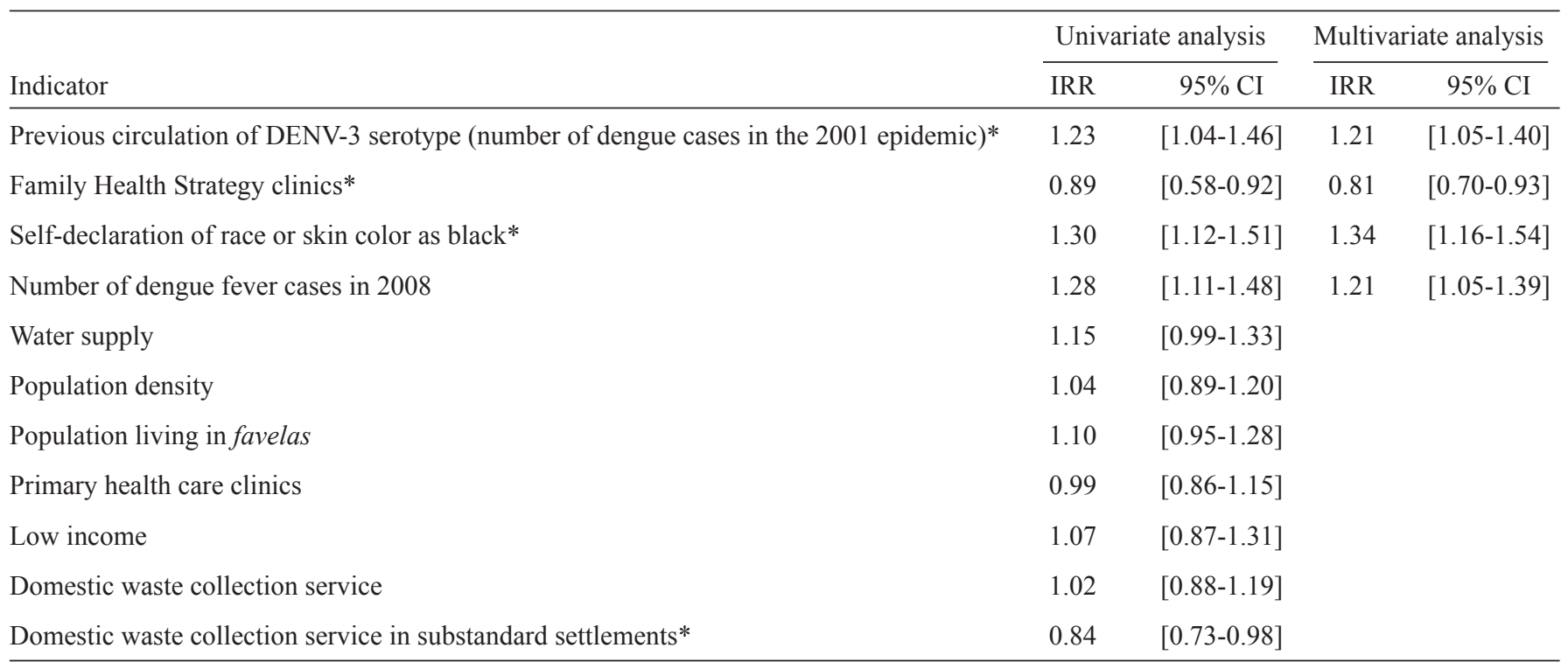

IRR: incidence rate ratio; 95\% CI: 95\% confidence interval; DENV-3: dengue virus serotype-3. *Variables with statistical significance in the univariate analysis; IRR reflects the variation in the incidence rate per each 1-standard-deviation increment in the mean of the predictive variable. The indicator domestic waste collection service in substandard settlements lost statistical significance as an explanatory variable in the multivariate analysis and was therefore eliminated from the final model.

TABLE 3 - Descriptive characteristics of 160 districts in the City of Rio de Janeiro, Brazil.

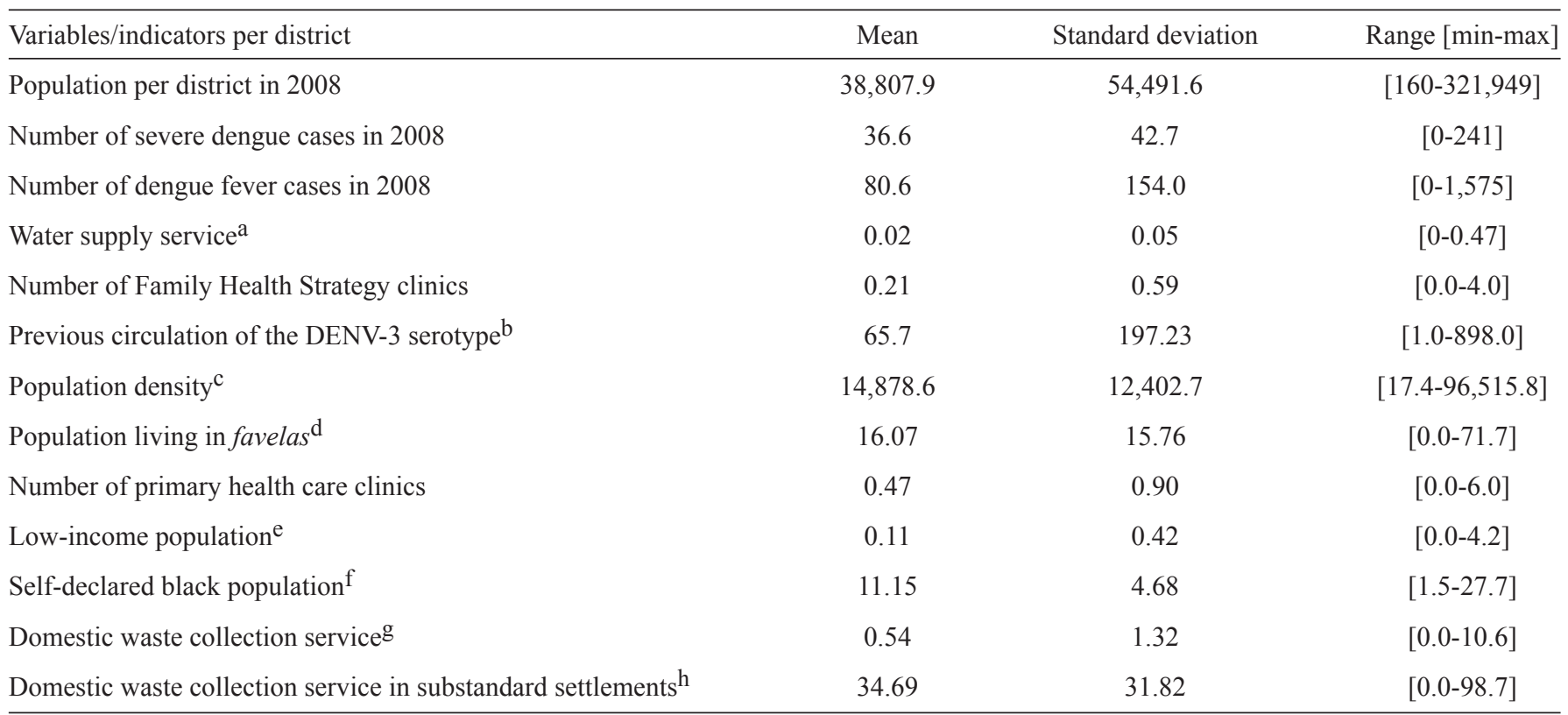

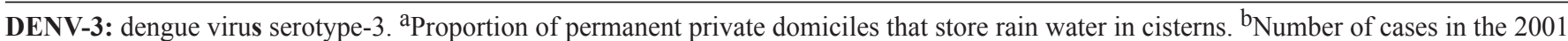
dengue epidemic. ${ }^{\mathrm{c}}$ Ratio of the district population to its area in $\mathrm{km}^{2}$. ${ }^{\mathrm{d}}$ Proportion of residents living in substandard settlements. ${ }^{\mathrm{e}}$ Proportion of residents in permanent private domiciles with no exclusive bathroom access and no toilet. fPercentage of residents who declared their skin color or race as black. ${ }^{\mathrm{g}}$ The proportion of permanent private domiciles that discharge domestic waste on empty lots or on the street. ${ }^{h_{\text {Proportion }}}$ of permanent domiciles in substandard settlements in which domestic waste is collected by open skip. 

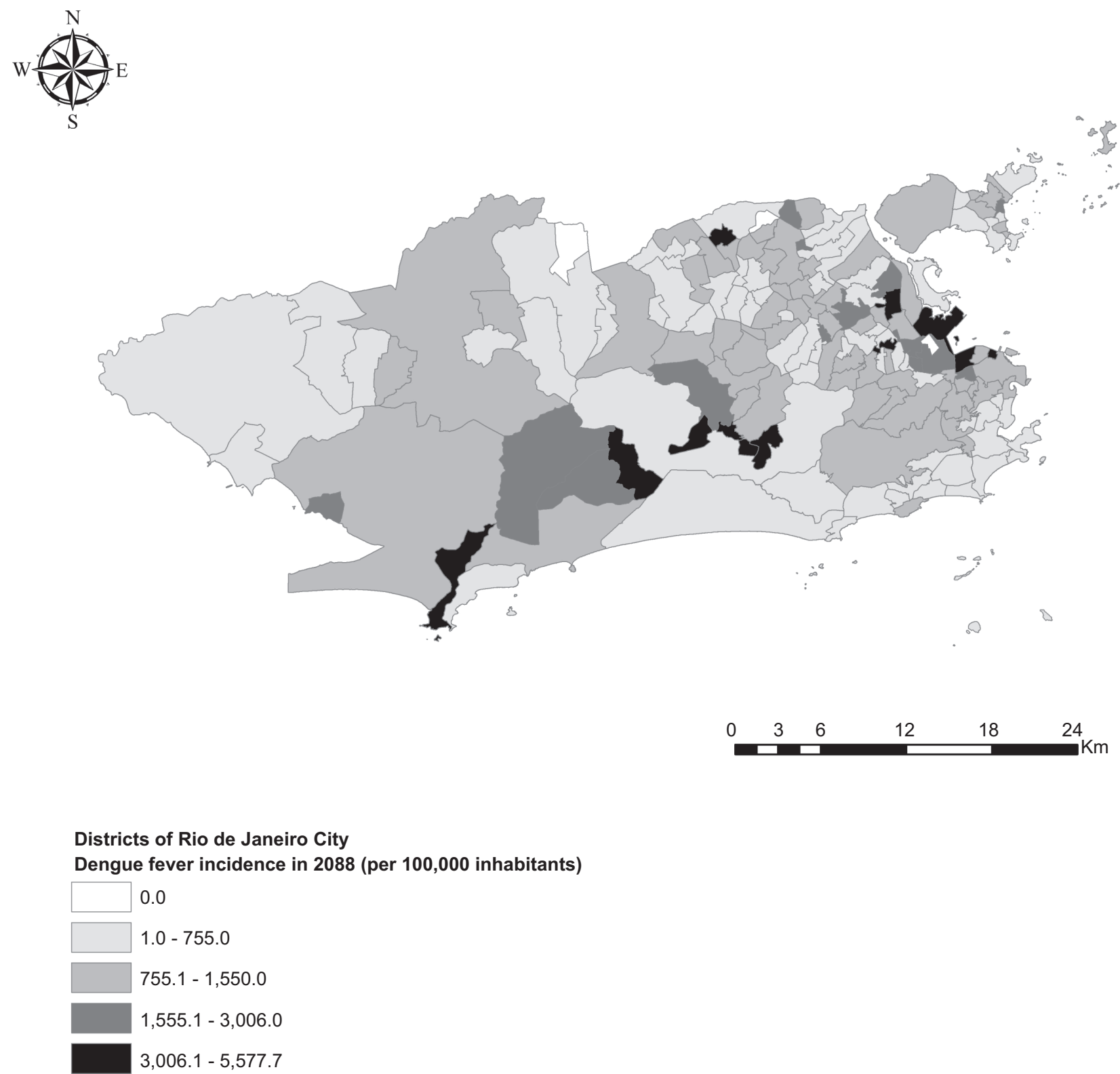

FIGURE 1 - Dengue fever incidence rate per district in Rio de Janeiro, Brazil in 2008.

Regarding the number of dengue cases in 2001 (considered to be a proxy for the prior circulation of the DENV-3 serotype), the districts presented an average of 65.7 cases with a range of 1-898 cases and a standard deviation of 197.2 cases (Table 3). Overall, the distribution pattern of dengue cases in 2001 suggests that cases were concentrated in the center, western and northern districts of the city.

The quartile with the highest percentage of self-declared black residents ranged from $14.7 \%-27.7 \%$ and comprised 38 districts. Notably, the overall mean percentage of self-declared black residents per district was $11.1 \%$ with a range of $1.5 \%$ $27.7 \%$ and a standard deviation of 1.3 (Table 3). The distribution pattern of self-declared black residents suggests that higher percentages of these individuals were located in the western and northern districts of the city.

The spatial distribution of the FHS clinics revealed only one district with four FHS clinics and one district with three clinics. Furthermore, six districts had two FHS clinics each, whereas 13 had only one clinic each. Therefore, of the 156 districts that were examined, 135 did not have FHS clinics. Both the location and quantity of FHS units are determined according to the population size, politicaladministrative factors and the local demand for health services.

Moran's coefficient for the residuals from the final multivariate model was not statistically significant ( $p$-value $=0.183$ ), indicating a lack of spatial correlation. 

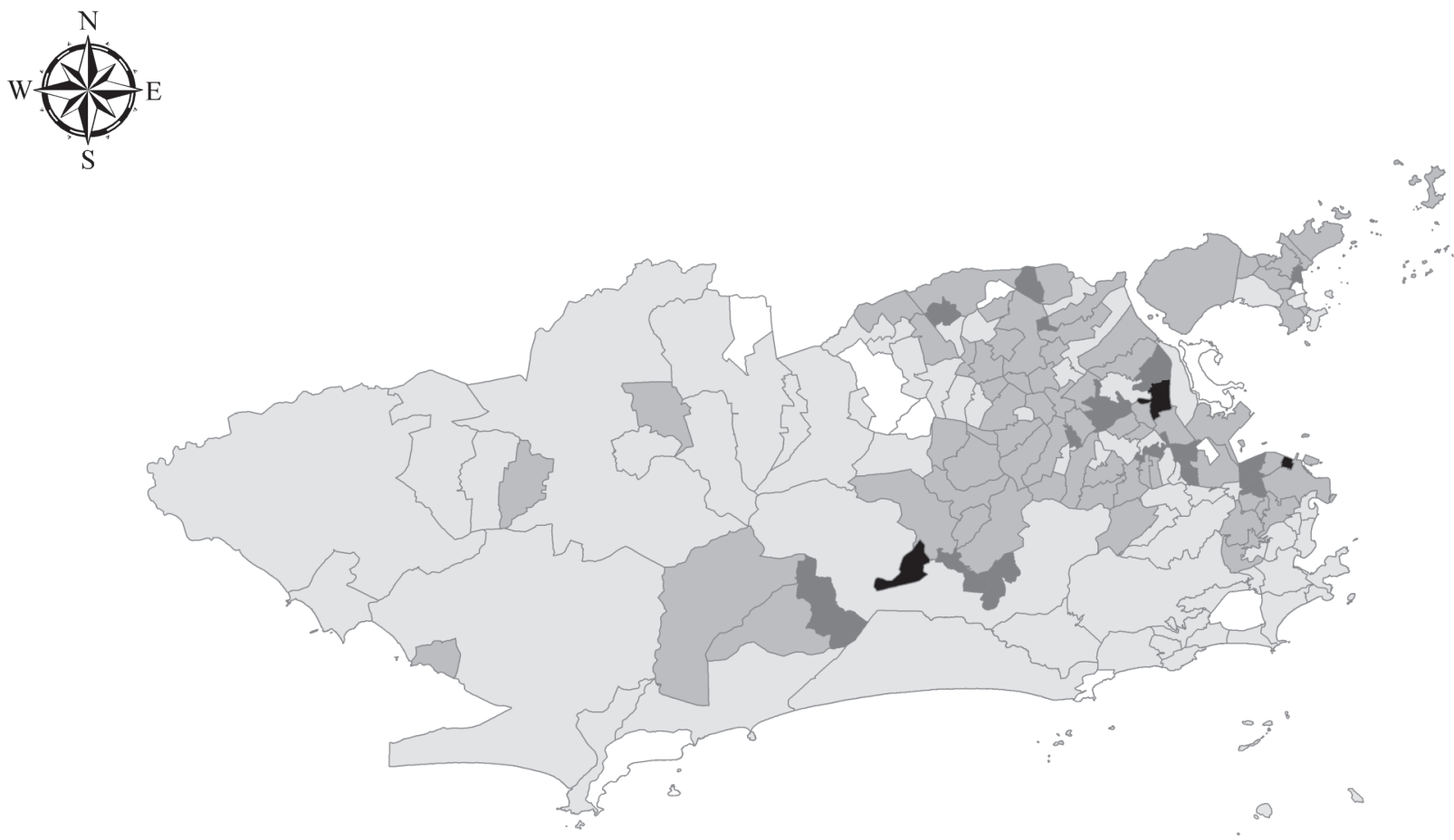

Districts of Rio de Janeiro City

Severe dengue incidence in 2008 (per 100,000 inhabitants)

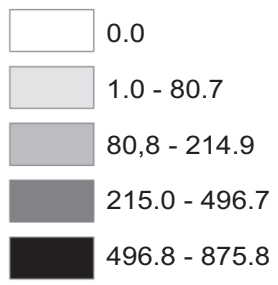

FIGURE 2 - Incidence rate of severe dengue per district in Rio de Janeiro, Brazil in 2008.

\section{DISCUSSION}

The higher incidence rates of severe dengue in 2008 in the districts with a higher incidence of dengue fever during the 2001 epidemic (attributed to DENV-3) suggests that more individuals were susceptible to secondary infection in these areas which may partially explain the substantial increase in severe cases, particularly in the 8- to 17-year-old age group. In 2008, 8- to 17-year-olds represented a large contingent of individuals who had been exposed to the DENV-3 serotype in the 2001 epidemic; however, these individuals were also susceptible to DENV-2 because they were born after the emergence of this serotype in 1990-19913,5.
Although several studies have demonstrated the occurrence of severe and fatal cases in primary dengue infection, sequential infection by multiple serotypes has been suggested as one of the main risk factors for the severe clinical evolution ${ }^{8,18}$. According to Barraquer et al. ${ }^{19}$, the accumulation of homotypic and heterotypic immunity against dengue virus serotypes in the Brazilian population since 1986 when dengue reemerged in Brazil created the conditions responsible for the changes that are now being observed in the clinical and epidemiological profiles of the disease. To the extent of a hyper-endemic scenario, adult individuals are acquiring new infections and accumulating heterotypic immunity to various serotypes.

However, when only severe cases of dengue (DHF and DSS), classified according to the WHO criteria, were used as 
the dependent variable in the regression analysis of this study, the explanatory variable confirmed dengue fever cases in 2001 lost statistical significance in the final multivariate model.

At the individual level, several genetics studies on the relationship between dengue severity and ethnicity/ancestry have demonstrated that afro-descendant individuals are less susceptible to the severe forms of the disease ${ }^{20,21}$. In contrast, at the ecological level, our findings indicate a higher risk of severe dengue in districts with a higher percentage of self-declared black residents.

This finding may be related to the historical socioeconomic vulnerability of this group, which in turn reflects the morbidity and mortality patterns of many health problems, including dengue and its severe forms ${ }^{22,23}$. These social inequalities may lead to several disadvantages, ranging from a lack of health promotion to a reduced capability to successfully treat severe and potentially fatal cases, resulting from limited access to early diagnosis and timely clinical management $\mathrm{t}^{23,24}$.

Most of the districts with a higher percentage of self-declared black residents have large areas of substandard settlements and favelas, which are generally associated with poor living conditions.

The national health system in Brazil is guided by the underlying principle of universal and egalitarian access to health care among socially disparate individuals; however, health inequities persist due to differences in living conditions and the availability and accessibility of health services ${ }^{22,25}$. Several authors have argued that income constitutes an enabling resource, whose availability positively influences health service access, i.e., the ability to use health services when necessary ${ }^{23,25,26}$.

Moreover, the characteristics of healthcare supply, such as availability and geographical location (proximity of health resources and services), can facilitate or hinder health service utilization $^{25}$. The results of this study demonstrate a protective effect against various forms of severe dengue in the districts with FHS clinics.

The introduction of the FHS was aimed at reorienting and strengthening the healthcare model in Brazil; therefore, the districts that have FHS clinics should have their health needs better met. Accordingly, ease of access to health care for individuals at the family level, in addition to preventive actions and health promotion, might have attenuated the vulnerability to dengue and its severe forms ${ }^{15}$.

Roriz-Cruz et al. ${ }^{14}$ emphasized the importance of structuring primary health care, especially the FHS, to achieve control of dengue in a comparison of Rio de Janeiro with its neighbor City, Niterói. They argue that although the cities are close and have similar profiles in terms of population density, climate and levels of sanitation, the incidence of dengue and related vector infestation rates are considerably lower in Niterói than in Rio de Janeiro. They attribute this difference to the high level of coverage by primary health care and the FHS, as the coverage in Niterói has increased from less than $1 \%$ to $77.4 \%$ over 20 years compared with $7.2 \%$ coverage in
Rio de Janeiro. Accordingly, the actions of community health workers at FHS clinics are directed at eliminating the foci of vectors and educating the community, which are critical factors to understanding the differences between the two cities in terms of the epidemiological profile of dengue ${ }^{14}$.

Most FHS clinics are not directly integrated with the dengue vector control program (Aedes aegypti); however, the actions of FHS are essential to health promotion and disease prevention, which includes reducing the risk of exposure to and transmission of dengue. Moreover, FHS teams are trained in early disease recognition, ensuring the referral of these patients to other care levels within the health system when necessary ${ }^{15,16}$.

This study had several limitations, such as the low rate of final classifications in the case records. The overloading of health care facilities, particularly during the dengue epidemic, may have contributed to information loss with respect to the classification of dengue cases. Therefore, even though severe cases tend to be recorded more carefully than are classic dengue cases, this issue should be considered while interpreting the results.

In conclusion, our results indicate the need for further studies to address the routine practices of the FHS with regard to dengue fever and its severe forms, including studies on the development of policies and procedures aimed at effective disease prevention from the perspective of primary health care at the local level.

\section{CONFLICT OF INTEREST}

The authors declare that there is no conflict of interest.

\section{FINANCIAL SUPPORT}

The authors would like to acknowledge the Foundation for Research Support in the State of Rio de Janeiro [Fundação de Amparo à Pesquisa do Rio de Janeiro (FAPERJ)] for financial support.

\section{REFERENCES}

1. World Health Organization (WHO). Special Program for Research and Training in Tropical Diseases (TDR). Dengue, guidelines for diagnosis, treatment, prevention and control. WHO/TDR; 2009. [Cited 2014 May 7]. Available at: http://www.who.int/tdr/ publications/documents/dengue-diagnosis.pdf/.

2. Gibson G, Souza-Santos R, Brasil P, Pacheco AG, Cruz OG, Honório NA, et al. From primary care to hospitalization: clinical warning signs of severe dengue fever in children and adolescents during an outbreak in Rio de Janeiro, Brazil. Cad Saude Publica 2013; 29:82-90.

3. Teixeira MG, Costa MCN, Coelho G, Barreto ML. Recent shift in age pattern of dengue hemorrhagic fever, Brazil. Emerg Infect Dis 2008; $14: 1673$.

4. Teixeira MG, Costa MCN, Barreto F, Barreto ML. Dengue: twentyfive years since reemergence in Brazil. Cad Saude Publica 2009; 25 (suppl 1): 7-18. 
5. Medronho RA. Dengue: increased severity and shift to childhood. Cad Saude Coletiva 2009; 17:301-304.

6. Nogueira RMR, Schatzmayr HG, Miagostovich MP, Farias MFDB, Farias Filho JC. Virological study of a dengue type 1 epidemic at Rio de Janeiro. Mem Inst Oswaldo Cruz 1988; 83:219-225.

7. Nogueira RMR, Miagostovich MP, Lampe E, Souza RW, Zagne SMO, Schatzmayr HG. Dengue epidemic in the state of Rio de Janeiro, Brazil, 1990-1: co-circulation of dengue 1 and dengue 2 serotypes. Epidemiol Infect 1993; 111:163-170.

8. Nogueira RMR, Miagostovich MP, Filippis AMB, Pereira MAS, Schatzmayr HG. Dengue virus type 3 in Rio de Janeiro, Brazil. Mem Inst Oswaldo Cruz 2001; 96:925-926.

9. Nogueira RM, Schatzmayr HG, Filippis AM, Santos FB, Cunha RV, Coelho JO, et al. Dengue virus type 3, Brazil, 2002. Emerg Infect Dis 2005; 11:1376-1381.

10. Giraldo D, Sant'Anna C, Périssé ARS, March MF, Souza AP, Mendes A, et al. Characteristics of children hospitalized with dengue fever in an outbreak in Rio de Janeiro, Brazil. Trans R Soc Trop Med Hyg 2011; 105:601-603.

11. Oliveira MF, Araújo JG, Ferreira Jr OC, Ferreira DF, Lima DB, Santos FB, et al. Two lineages of dengue virus type 2, Brazil. Emerg Infect Dis 2010; 16:576-578.

12. Ministério da Saúde do Brasil (MS). Secretaria de Vigilância em Saúde. Guia de Vigilância Epidemiológica 2005. $6^{\mathrm{a}}$ edição, Brasília (DF): MS. [Cited 2014 May 7]. Available at: ftp://ftp.cve.saude. sp.gov.br/doc_tec/ZOO/lepto_gve7ed_atual.pdf/.

13. Roriz-Cruz M, Sprinz E, Rosset I, Goldani L, Teixeira MG. Dengue and primary care: a tale of two cities. Bull World Health Organ 2010; 88:244.

14. Neto FC, Barbosa AAC, Cesarino MB, Favaro EA, Mondini A, Ferraz AA, et al. Dengue control in an urban area of Brazil: impact of the Family Health Program on traditional control. Cad Saude Publica 2006; 22:987-997.

15. Fernandes LCL, Bertoldi AD, Barros AJD. Health service use in a population covered by the Estratégia de Saúde da Família (Family Health Strategy). Rev Saude Publica 2009; 43:595-603.
16. Myers WP, Westenhouse JL, Flood J, Riley LW. An ecological study of tuberculosis transmission in California. Am J Public Health 2006; 96:685-690.

17. Byers AL, Allore H, Gill TM, Peduzzi PN. Application of negative binomial modeling for discrete outcomes: a case study in aging research. J Clin Epidemiol 2003; 56:559-564.

18. Jain A, Chaturvedi UC. Dengue in infants: an overview. FEMS Immunol Med Microbiol 2010; 59:119-130.

19. Barraquer IR, Cordeiro MT, Braga C, Souza WV, Marques ET, Cummings DAT. From re-emergence to hyperendemicity: the natural history of the dengue epidemic in Brazil. PLoS Negl Trop Dis 2011; 5:e935.

20. Blanton RE, Silva LK, Morato VG, Parrato AR, Dias JP, Melo $\mathrm{PR}$, et al. Genetic ancestry and income are associated with dengue hemorrhagic fever in a highly admixed population. Eur J Hum Genet 2008; 16:762-765.

21. Sierra BC, Kourí G, Guzman MG. Race: a risk factor for dengue hemorrhagic fever. Arch Virol 2007; 152:533-542.

22. Victoria CG, Matijasevich A, Silveira MF, Santos IS, Barros AJD, Barros FC. Socio-economic and ethnic group inequities in antenatal care quality in the public and private sector in Brazil. Health Policy Plan 2010; 25:253-261.

23. Barata RB, Almeida MF, Montero CV, Silva ZP. Health inequalities based on ethnicity in individuals aged 15 to 64, Brazil, 1998. Cad Saude Publica 2007; 23:305-313.

24. Figueiró AN, Hartz ZMA, Brito CAA, Samico I, Filha NTS, Cazarin $\mathrm{G}$, et al. Óbito por dengue como evento sentinela para avaliação da qualidade da assistência: estudo de caso em dois municípios da Região Nordeste, Brasil. Cad Saude Publica 2011; 27:2373-2385.

25. Travassos C, Oliveira EXG, Viacava F. Geographic and social inequalities in the access to health services in Brazil: 1998 and 2003. Ciênc Saude Coletiva 2006; 11:975-986.

26. Anderson RM. Revisiting the behavioral model and access to medical care: does it matter? J Health Social Behav 1995; 36:1-10. 